\title{
Secure e-health insurance model
}

\author{
${ }^{1}$ G.V. Ramesh Babu, ${ }^{2}$ Prof. M. Padmavathamma \\ Department of computer science, S.V. University Tirupati, A.P. India
}

\begin{abstract}
In the current era of modern health problems should be addressed with utmost importance and data should be secured in the competitive market in order to avoid misuse by third parties which are not responsible in this regard. In view of Health insurance which benefit patients economically should be effective and should also address the issue of providing privacy to their medical record. To address the above issues we are proposing architecture new secure e-health issue system (SEHIS) model which allows to store patients data in Smart e-Health Card (SEHC) which is given to patients by Health Insurance companies in turn used by medical practitioners to access patients data. SEHIS provides expert services to advice patients regarding insurance schemes selection (decision Support system) verify and secure patients data in SEHC. In this model, SEHC contains patients data like finger prints, photo and personnel data is encrypted by using PKI, Digital Image encryption techniques. SEHIS model verifies counterfeit documents, if any of the patient to use insurance facility or an insured person and a physician may deviate from insurance company rules to get benefits illegally etc. Iin our proposed model, SEHIS will benefit insurance companies, patients and works accordance with HIPPA requirements.
\end{abstract}

\section{Introduction}

Enforcing of information technology in Health Care System is essential it needs to make effective service oriented to the clients. In which, health care services like e-Insurance Health Care System is used to diagnosis through Doctor authority in order to get benefit from the heath area. In which, expert system in health care service for health insurance patient clients for better medical services. In the area of health care system for diagnosis and deals with expert system model which allows diagnosis of diseases and an expert evaluate the diagnosis processed by the system in Doctor authority diagnosis several diseases. It maintains information from past history and symptoms.

Security for e-Health Insurance has vital aspect. The authentication of patient who utilize the benefit of health insurance. In the e-Health Insurance field Digital Health Card which benefit to the patient for treatment. In which patient can communicate their health information through web service. In this process, image encryption can be maintained on patient photo, details and test reports. Insured patient is utilize through hospitals for treatment in which Doctor authority connect through web service of entering into the particular site for medical operations through expert verification system such as, Diagnosing etc., [5,6].

To verify client requirements for expert verification system is normally performed by testing. In this model SEHC contains patients data like finger prints, photo and personnel data is encrypted by using PKI, Digital Image encryption techniques. SEHIS model verifies counterfeit documents, if any of the patient to use insurance facility or an insured person and a physician may deviate from insurance company rules to get benefits illegally etc. in our proposed model SEHIS will benefit insurance companies, patients and works accordance with HIPPA requirements.

\section{Related Work}

\subsection{Digital Health Card System:}

Smart Cards are efficient for security for authentication smart card which interconnect health care providers it is used for patient authentication and doctor authority for medical operations. To secure sensitive information electronic implementation of Digital Health Card and security interoperability like cryptography standards besides, to ensure authenticity of health card. Digital Health Card is used to maintain authentication it has data storage and processing capabilities. In a online based health care system individual data is kept in patient health card.[7]

\subsection{Expert System:}

Expert system occurs to facilitate in different filed or areas such as medical. In which one of the prime areas i.e., medical analysis through decision making. Expert systems normally make different stages such as analysis and diagnosis through which patient status can be determined. Expert system diagnosis and suggest about particular diseases knowledge based management system. Expert system are a branch of Artificial Intelligence (AI) and were developed by the AI Community in the mid-1960s. an expert system can be defined 
as "an intelligent computer program that uses knowledge and inference procedures to solve problems that are difficult enough to require significant human expertise for their solutions". Expert system have several applications in various domains. They are mostly relevant in order to develop an expert system the knowledge is converted into a computer programme. Knowledge is represented in the form of IF-THEN rules. Health care professionals such as doctors, clinicians, pharmacist, diagnosis and treatment can be made through web service depending upon the data available in the patient details. [8]

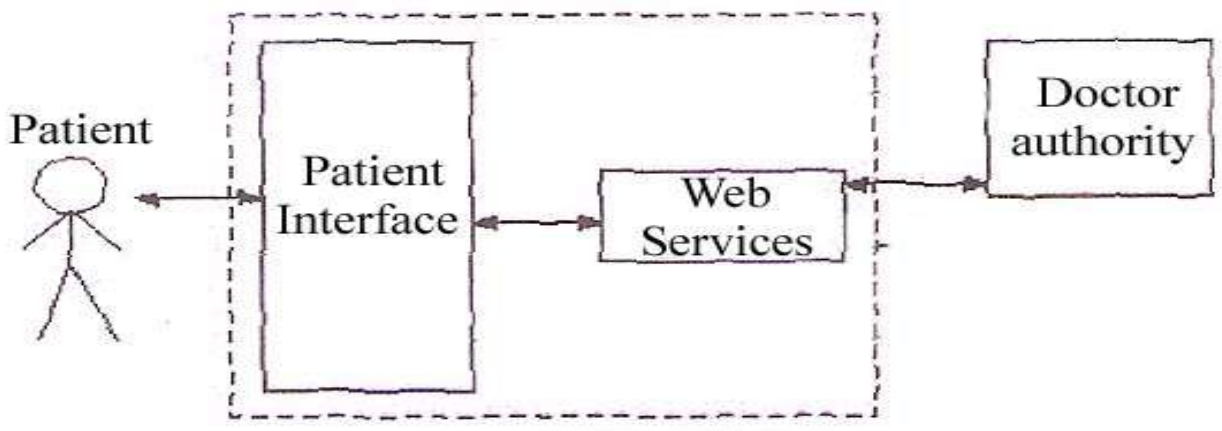

\subsection{Image encryption Fig. 1: Expert System}

Image encryption process transforms plain - image information into cipher-image for involving the original image with one or more key. Technology that use the same secret key for encryption and decryption under private key techniques asymmetric key technique use two different keys, are public key for encryption and two private keys for decryption. Cryptosystem can be serve all types of attacks they try to violate the system such as, Plain test attack, cipher text attack.[7,8]

\section{Encryption image:}

1. Load the plain image (original image)

2. Calculate the width and height of the input image.

3. Lower horizontal number of blocks $=$ integer $($ Image - Height $/ \mathrm{n})$

4. Lower vertical number of Blocks $=$ integer $($ Image $=$ width $/ \mathrm{n})$

No of blocks $=$ horizontal number of blocks $\mathrm{x}$ vertical number of blocks[7]

\subsection{Biometric authentication:}

Biometric authentication can be maintained by using finger printing.

\section{Methodolgy}

First and foremost client selects the scheme of insurance. After scheme selection client needs to register and furthermore, payments is to be paid. While process stars verification through insurance authority to be performed. After verification process completed Digital Health Card generated to the client. Then client patient submits his health card to the doctor authority. In the doctor authority diagnosis based on symptoms based on lab test reports. During the transaction process secure communication essential between patient client and doctor authority through encrypted of image. In this scenario authentication and confidentiality are major concern to ensure security..

\section{Proposed Architecture}

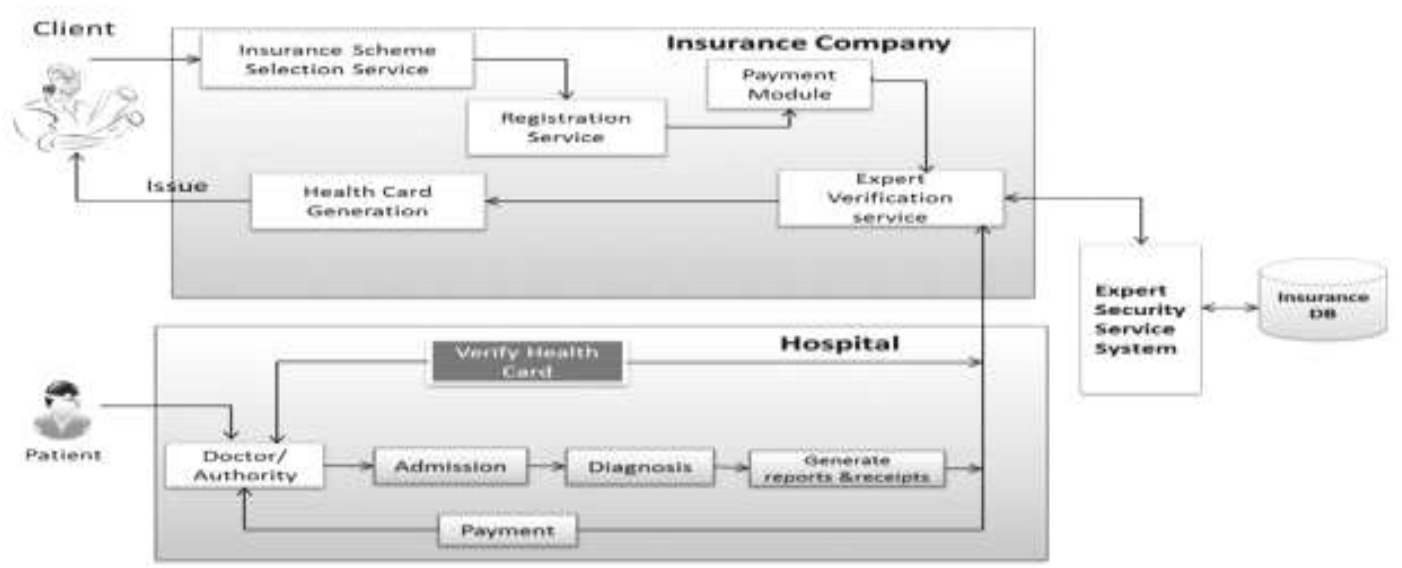

Fig.2: Proposed Architecture 


\section{Conclusion}

In this paper, we have developed architecture for e- health insurance system which serve patient communications with doctor authorities.

\section{References}

[1] "New variant M. J2 - RSA Cryptosystem" : E. Madhusudhan Reddy, B. H. Nagaraiasri, A. B. Rajesh kumar, M. Padmavathamma.

[2] "Threshold Extended ID3 algorithm", A.B. Rajesh Kumar, C. Pani Ramesh, E. Madhusudhan, M. Padmavathamma, ICDIP 2012 International Conference in Malasia.

[3] "A cryptographic key management solution for HIPAA privacy/ security regulations: Wei - Bin Lee, Member, IEEE, and Chien Ding Lee, IEEE transaction on information technology in biomedicine, vol 12, No.1, January 2008.

[4] Image encryption using block - based transformation algorithm:Mohammad Ali Bani Younes and Aman Jantan IAENG international journal of computer science, 35: 1, IJCS 35_1_03.

[5] Design and implementation of a smart card based healthcare information system: Geylani Karda, E. Turhan Tunali* Computer methods and programs in biomedicine 8I(2006) 66-78.

[6] A novel encryption method for image security: Mohammed Abbas Fadhil A1- Husainy, internation journal of security and its applications Vol. 6 No. 1 January, 2012.

[7] "Secure E-Health Care Model” IOSR Journal of Computer Engineering (IOSRJCE) ISSN: 2278-0661, ISBN: 2278-8727 Volume X, Issue X (Sep-Oct. 2012), PP 00-00

[8] Rule-based Expert System for Diagnosis and Symptom of Neurological Disorders "Neurologist Expert System (NES)" ICCIT 2012. 